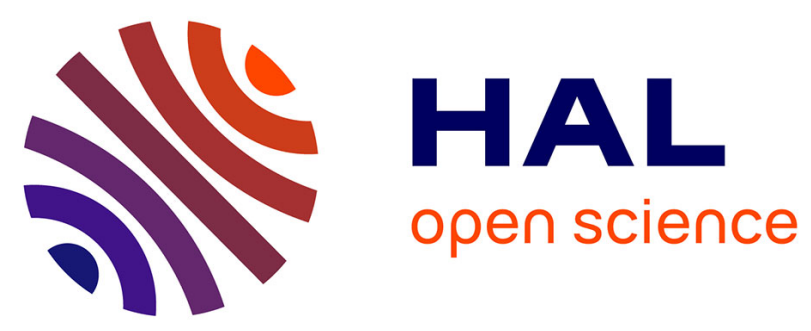

\title{
Study of Electrode Locations for Joint Acquisition of Impedance- and Electro-cardiography Signals
}

Margus Metshein, Antoine Gautier, Benoit Larras, Antoine Frappé, Deepu John, Barry Cardiff, Paul Annus, Raul Land, Olev Martens

\section{To cite this version:}

Margus Metshein, Antoine Gautier, Benoit Larras, Antoine Frappé, Deepu John, et al.. Study of Electrode Locations for Joint Acquisition of Impedance- and Electro-cardiography Signals. 2021 43rd Annual International Conference of the IEEE Engineering in Medicine \& Biology Society (EMBC), Nov 2021, Mexico, Mexico. 10.1109/EMBC46164.2021.9629504 . hal-03482302

\section{HAL Id: hal-03482302 https://hal.science/hal-03482302}

Submitted on 17 Dec 2021

HAL is a multi-disciplinary open access archive for the deposit and dissemination of scientific research documents, whether they are published or not. The documents may come from teaching and research institutions in France or abroad, or from public or private research centers.
L'archive ouverte pluridisciplinaire HAL, est destinée au dépôt et à la diffusion de documents scientifiques de niveau recherche, publiés ou non, émanant des établissements d'enseignement et de recherche français ou étrangers, des laboratoires publics ou privés. 


\title{
Study of Electrode Locations for Joint Acquisition of the Impedance- and Electro-cardiography Signals
}

\author{
Margus Metshein ${ }^{1}$, Antoine Gautier ${ }^{2}$, Benoit Larras ${ }^{2}$, Antoine Frappe ${ }^{2}$, Deepu John ${ }^{3}$, Barry Cardiff ${ }^{3}$, \\ Paul Annus ${ }^{1}$, Raul Land ${ }^{1}$, and Olev Martens ${ }^{1}$
}

\begin{abstract}
ICG (impedance cardiography) and ECG (electrocardiography) are important indicators of the functioning of the heart and of the overall cardio-vascular system. Adding of the ICG to ECG measurement functions into the wearable devices will improve the quality of the health monitoring of the people, as the ICG is reflecting relevant hemodynamic parameters (informative time intervals, but also the stroke volume and cardiac output and their variability). In the current paper various electrode locations (12 various setups) has been tested for possible joint ECG \& ICG data acquisition (by the same electrodes) and the quality has been evaluated for every setup. It is shown, that while typically the ICG is acquired over the whole thorax (or the chest), the wrist-based joint acquisition of the ECG \& ICG signals can be considered with still reasonable and acceptable signal quality.
\end{abstract}

\section{INTRODUCTION}

For monitoring the pulse, which is among blood pressure, temperature, height and weight, the most important parameters of human body, classical methods exist. The methods for evaluating the status of heart are available already for a long time - from which the most known is electrocardiography. During the development of technology, also the impedance cardiography (ICG) has been emerged - capable of assessing the status of cardiovascular system. Importantly, the electrocardiogram (ECG) presents the electrical activity of the heart, while ICG denotes the mechanical pumping activity of the heart [1] - representing the same information in different modalities. Both methods are well researched, relying on classical and predefined placement of electrodes. The co-utilization of both methods had opened a door to determine specific time intervals, characterizing the overall operating of heart, relying on both, mechanical and electrical functioning of heart.

ICG is a known method for determining hemodynamical parameters and has been used from 1960-s [2] to estimate additionally the heart rate (HR) and its variation (HRV), the Cardiac Output (CO) and Stroke Volume (SV), but also various timings, e.g., Left Ventricular Ejection Time (LVET) and Pre-Ejection Period (PEP). Portable solutions can be

\footnotetext{
* This work was supported by EU Regional Development Fund (Estonian Centre of Excellence in ICT Research EXCITE TAR16013), CHIST-ERA grant JEDAI, Mobilitas+ project Mobera20, Irish Research Council and French Researh Agency project ANR-19-CHR3-0005-01.

1 M. Metshein, P. Annus, R. Land and O. Martens are with Tallinn University of Technology, Estonia (e-mail: margus.metshein@ @altech.ee).

2 A. Gautier, B. Larras and A. Frappe are with Univ. Lille, CNRS, Centrale Lille, Junia, Univ. Polytechnique Hauts-de-France, UMR 8520 IEMN, Lille, France.

${ }^{3}$ D. John and B. Cardiff are with University College Dublin, Ireland.
}

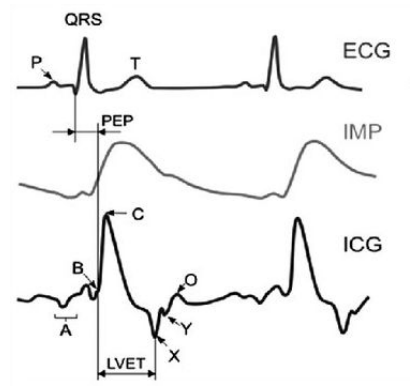

Fig. 1. Typical ECG and ICG waveforms [3]

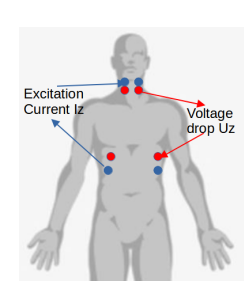

(a) Classical ICG measurement setup

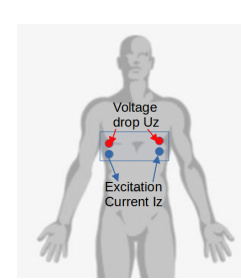

(b) Wearable belt based ICG measurement setup

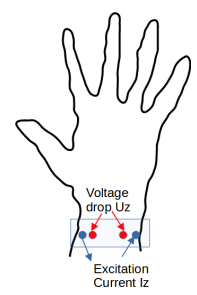

(c) Wrist EBI measurement setup
Fig. 2. Various ICG measurement setup configurations

developed to monitor these and other hemodynamic's related parameters [3].

For measuring ECG, the standard lead locations are defined, from which the Einthoven's triangle have been known already 120 years. The elaboration of the electrode placement strategies has occurred since then with the goal of gaining fully complex and detailed signal where the prerequisite is the location of heart in the center of the triangle. The recognizable pattern of ECG signal is classically identified by assigning five deflections in the detected signal: $\mathrm{P}, \mathrm{Q}, \mathrm{R}$, $\mathrm{S}$ and $\mathrm{T}$ wave.

Example waveforms of ECG and ICG are given in Fig. 1. As described below and illustrated in the Fig. 2a, the ICG (as well as ECG) signals are classically acquired from the whole thorax. However, the human body is a complex entity, providing option to acquire the relevant data also from the chest (Fig. 2b) or even from the wrist (Fig. 2c).

The simplest way of monitoring hemodynamic parameters is detecting the pulse. This is the oldest method, rebuilt in modern devices through different means, like electrical bioimpedance (EBI), bioelectricity (ECG), optical (photoplethysmography (PPG)) etc. based methods. However, such signals are sophisticated, presenting variety of key points that 
represent the operating of heart.

However, the classical electrode locations for monitoring ICG and ECG are clearly causing the devices to be bulky or very complex. To reduce the size of the device or utilized area on the body surface, new electrode locations need to be chosen - presumably located more densely. This, in turn, is expected to lower the quality of the measured signals, cause these to contain less information or to be nonlinear compared to the actual stroke volume and measured value of EBI.

Based on its noninvasive means, ICG fits perfectly into the framework of the term: wearability - the incorporation of electrodes and respective electronic circuitry into smart wearables for on-site patient monitoring. For monitoring ICG, electrodes are attached onto the skin surface, current of high frequency and low amplitude is applied onto the thorax and resulting response voltage is recorded. The resulting voltage is amplitude-modulated, incorporating the data of pulsatile changes in the impedance of thorax, expectedly caused by cardiorespiratory system [4].

Wrist is often proposed as a location of modern wearable devices - solved classically through touching the electrode of smartwatch with a finger of other hand (like Apple Watch). Also, the applicability of single-arm singe-lead monitoring of ECG has been reported in scientific literature [2], [5], capable of replicating all the key components. However, the signal amplitude is significantly reduced with the diminished volume of body between the electrodes [6], requiring heavy filtering or advanced denoising strategies.

The co-utilization of electrodes for monitoring simultaneously the ICG and ECG have been reported in literature before. For example [7], where the number of electrodes for wearable autonomous measurement device has been reduced to four with the recognizability of hemodynamic feature points in both signals. Or a patented solution for monitoring ICG and ECG with the configuration where the electrodes are shared [8]. In laboratory environment, such experiments have been reported, incorporating concurrent utilization of variety of sensors - like in [9], where the ICG and ECG is detected by using the classical Kubicek method (using band electrodes around the thorax).

However, the recognizability of the hemodynamic pattern on the signal is expected to deteriorate when relocating the electrodes from the nominated to novel positions. Due to that, the determination of feature points in ICG and ECG, gathered by using coinciding electrode location in the arm has rarely been referred in relevant literature, constituting a novel research direction. Still, it is expected that the hemodynamic pattern on the monitored ICG can be determined - even in the case of non-classical electrode locations. Current paper studies the possibility of co-utilizing the coinciding electrode locations for monitoring ECG and ICG. The detection of hemodynamic pattern in ICG and time intervals parallel with ECG is performed, solutions proposed, and various electrode placements are evaluated.

\section{Methods and Measurement Setup}

\section{A. Measurement Devices and Properties}

The impedance spectroscope HF2IS, accompanied with transimpedance amplifier HF2TA of Zurich Instruments AG (Zurich, Switzerland) was used as a measurement device. HF2IS approves performing simultaneous measurement of impedance at four distinct frequencies up to $50 \mathrm{MHz}$ [10], with two optional auxiliary inputs, one of them connected to an external ECG monitor for concurrent acquisition of respective analog signals. The measurement frequency (typical for ICG) of $100 \mathrm{kHz}$ was selected in this study.

A custom prepared ECG monitor with 3-electrode system was used to gather the data of electrical activity of heart. The ECG monitor was based on the single lead heart rate monitor front end of type AD8232ACPZ of Analog Devices Inc. (Wilmington, MA, USA).

For measurement of ICG and ECG, monitoring electrodes of type 2228 of 3M (Maplewood, MN, USA) were used, chosen because of its firm adhesive foam tape backing.

\section{B. Measurement Method}

The experimentation with electrode locations for measuring the ICG and ECG was divided into two domains: placed either on the thoracic area (1) or exclusively on the left arm (2). The possibility of utilizing the same electrode locations for determining the ICG and ECG was researched in both cases. Physically separate electrodes were used to pick up ICG and ECG - attached side by side on the skin surface. This approach is explained by the utilization of the single impedance spectroscope without any additional circuitry of signal pick-up - needed for co-usage of the same electrodes. As the electrodes were closely attached, the outcome is comparable, and can be considered to imitate the situation when the same electrodes are used.

For implementing the classical Einthoven's triangle, three leads are specified. However, current research was focused on using single lead positive left arm (LA) electrode, right arm negative (RA) electrode approach with the attached third left leg (LL) electrode.

The electrodes for ECG and ICG on the left arm were not located as a pair, but had larger distance in the case of ECG. Specifically, LA electrode was located on the upper arm, while RA together with LL were attached onto wrist. The reason for this is the deterioration of the quality of detected signal in the case of single-arm ECG [5].

To keep the descriptions homogeneous throughout the whole paper, the term ICG is used to remark the electrode placement configurations on both cases (thorax and arm): called thoracic electrode placement configurations (TEPC) and arm electrode placement configurations (AEPC) respectively.

The electrodes were attached onto the skin surface based on the chosen TEPC's and AEPC's and after 3 minutes, the signal waveforms of ECG and ICG were picked up. The electrodes were attached to the impedance spectroscope by using wires with crocodile clips that were implemented to be as short as possible. 


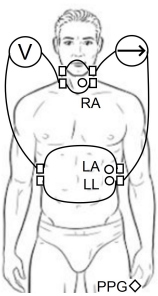

(a) TEPC-1

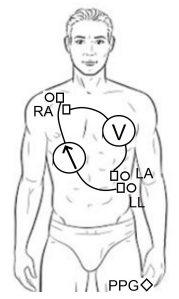

(b) TEPC-2

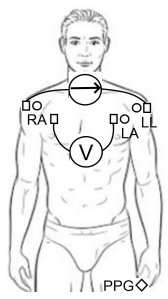

(c) TEPC-3

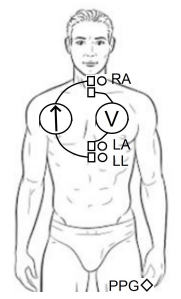

(d) TEPC-4
Fig. 3. Measurement configuration setups: TEPC-1 to TEPC-4

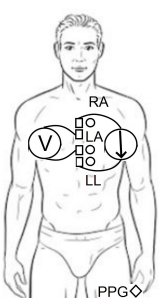

(a) TEPC-5

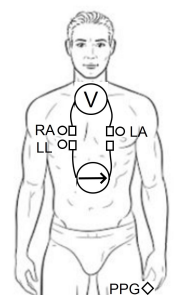

(b) TEPC-6

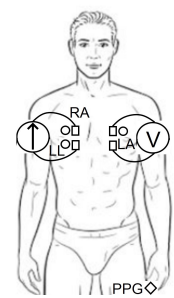

(c) TEPC-7

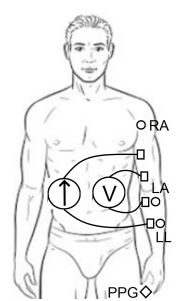

(d) AEPC-5

(Thorax base)
Fig. 4. Measurement configuration setups: TEPC-5 to TEPC-7 and AEPC-5

The experimental procedures involving human, described in current paper were following the principles outlined in the Helsinki Declaration of 1975, as revised in 2000.

\section{Setup for Monitoring ECG and ICG on the Thoracic Area}

For monitoring ICG on the thoracic area, seven setups were chosen for TEPC. The configurations were either following the classical ICG monitoring electrode locations like TEPC-1 [11] (Fig. 3a); or repeating a literature-based proposal of co-monitoring ICG and ECG like TEPC-2 [7] (Fig. 3b); or intuitive TEPC-3 and TEPC-4 (Fig. 3c-d), where the heart or aorta remains between the electrodes; or presenting new compact approaches that could be suitable for incorporation into a wearable device by forming TEPC-5 to TEPC-7 (Fig. 4a-c).

\section{Setup for Monitoring ICG and ECG on the Left Arm}

For monitoring ICG on the left arm, five AEPC variants were selected (Fig. 5). Electrodes were either attached distally like AEPC-1; or circularly like AEPC-2; or in mixed configuration respective to the wrist like AEPC-3 and AEPC4 ; or covering the whole length of arm like AEPC-5. In the case of AEPC-1 to AEPC-4, the original size of electrodes was cut to the width of $5 \mathrm{~mm}$ to gain higher electrode density.

\section{Measurement Results and Initial Evaluation}

The Impedance $(\mathrm{Z})$ and ECG signals were acquired from a young healthy male (37 years of age) and saved in the

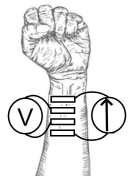

(a) AEPC1

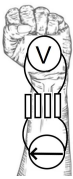

(b) AEPC2

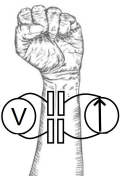

(c) AEPC3

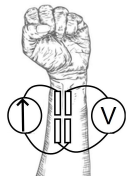

(d) AEPC4
Fig. 5. Measurement configuration setups: AEPC-1 - AEPC-5

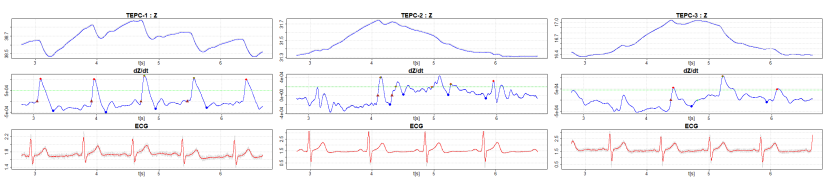

(a) TEPC-1

(b) TEPC-2

(c) TEPC-3

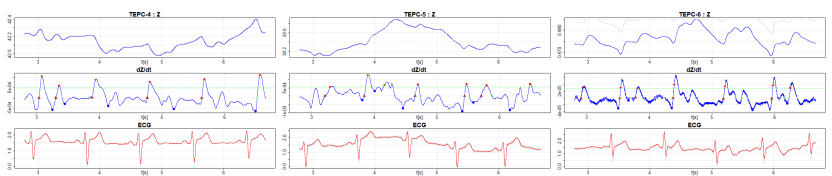

(d) TEPC-4

(e) TEPC-5

(f) TEPC-6

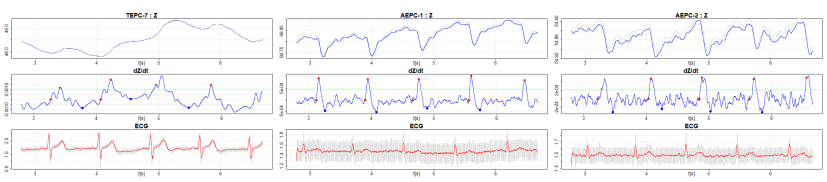

(g) TEPC-7

(h) AEPC-1

(i) $\mathrm{AEPC}-2$

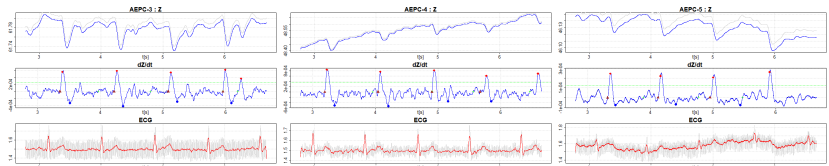

(j) AEPC-3

(k) AEPC-4

(1) AEPC-5

Fig. 6. Measurement results of Z, ICG and ECG for various setups, shown in Ohms for Z and dZ/dt and in Ohms for ECG

data logs. Single data log was selected for every (of 12) case from series of 3 measurements (after visually compared the $\mathrm{dZ} / \mathrm{dt}$ and ECG graphs, to have more-or-less of similar quality as not all the signals in the sets of three were usable). A Savitzky-Golay low-pass filter was applied on the $\mathrm{Z}$ signal (sampling frequency $-1500 \mathrm{~Hz}$, filter order - 5, number of points - 49) before differentiation. The feature points $\mathrm{B}, \mathrm{C}$, $\mathrm{X}$ (Fig. 1) were determined by a very simple algorithm [12]: $\mathrm{C}$ as maximum (extremum over ca $50 \%$ of the peak value threshold), B as the first zero-crossing to the "left" from the $\mathrm{C}$ and $\mathrm{X}$ as the global minimum between two $\mathrm{C}$ points.

The Z, dZ/dt and ECG with estimated B, C, X points of ICG (on dZ/dt plots) are shown in Fig. 6 for all described measurement configurations. In the Fig. 6, Z and dZ/dt are shown in Ohms and the ECG in Volts.

The recognizability of these key points depends largely on the electrode placement on the body. The classical electrode placements have been proposed to provide the most sophisticated signal waveforms as possible - like the modified version of Kubicek method, proposed by Sramek [11] based on spot electrodes. In such methods, the electrodes are attached to cover the whole volume of upper thorax, including the heart and aorta. Other, either tightly set or special EPC's may not cover the whole interesting volume changes in thorax and the waveforms of ICG may be less informative, need additional processing or smart analysis. The presence of feature points in the signal of ICG can be used as a base to evaluate the utilized EPC's.

In the current paper the focus was on determining the $\mathrm{B}, \mathrm{C}$, 
TABLE I

QUALITY ESTIMATION OF THE MEASURED SIGNALS OF ICG: 0,1 - NOT ACCEPTABLE; 2,3-ACCEPTABLE

\begin{tabular}{lllllll}
\hline No & Conf & Quality & $\begin{array}{l}\text { C-C } \\
\text { errors }\end{array}$ & $\begin{array}{l}\text { C-X } \\
\text { errors }\end{array}$ & $\begin{array}{l}\text { B-C } \\
\text { errors }\end{array}$ & $\begin{array}{l}\text { Total } \\
\text { periods }\end{array}$ \\
\hline 1 & TEPC-1 & $\mathbf{3}$ & 0 & 0 & 0 & 16 \\
2 & TEPC-2 & 1 & 9 & 9 & 8 & 16 \\
3 & TEPC-3 & 1 & 2 & 6 & 3 & 9 \\
4 & TEPC-4 & 2 & 0 & 0 & 0 & 15 \\
5 & TEPC-5 & 1 & 3 & 9 & 1 & 13 \\
6 & TEPC-6 & 0 & 11 & 16 & 16 & 27 \\
7 & TEPC-7 & 2 & 2 & 12 & 6 & 12 \\
8 & AEPC-5 & 2 & 0 & 8 & 2 & 17 \\
9 & AEPC-1 & 3 & 0 & 4 & 0 & 17 \\
10 & AEPC-2 & 2 & 2 & 7 & 0 & 18 \\
11 & AEPC-3 & 2 & 2 & 9 & 0 & 18 \\
12 & AEPC-4 & 2 & 0 & 6 & 0 & 17 \\
\hline
\end{tabular}

$\mathrm{X}$ points of the ICG, needed to estimate the most important LVET, SV and CO values of hemodynamics.

\section{Discussion And Quality Estimation of The ICG SIGNALS}

While the ECG signal is reasonable for all studied cases (see plots in Fig. 6), then the ICG (dZ/dt) signal has quite varying quality. The goodness of ICG signals is estimated in two ways (column "Quality" (1) and columns "C-C errors", "C-X errors" and "B-C errors" (2) in Table I). First, the plots with marked possible B, C, X points are evaluated visually 0 (non-recognizable waveform); 1 (waveform visible, but not possible to find the feature points); 2 (reasonable); 3 (strong and clear signal). These terms were chosen in the frames of conducted research, where the signals starting from the 2 (reasonable) denote the situation where the algorithm was capable of detecting the feature points.

Secondly the estimated locations of $\mathrm{B}, \mathrm{C}, \mathrm{X}$ points, the time intervals $\mathrm{C}-\mathrm{C}$ (cardiac period), B-C and C-X (forming together the LVET) were estimated and compared against expected values around - $900 \mathrm{~ms}(\mathrm{C}-\mathrm{C}), 60 \mathrm{~ms}(\mathrm{~B}-\mathrm{C})$ and $200 \mathrm{~ms}(\mathrm{C}-\mathrm{X})$. The count of these erroneous time intervals against the overall number of periods is shown in the same table. The reasonable setups are (in green) - TEPC-1, 4, 7 and AEPC-1 to AEPC-5. As an observation for future work, while the AEPC-s can be as good as TEPC-s, the C-X interval for AEPC can be with error of 30 or $50 \%$ or even more, probably due to the wrong determination of the $\mathrm{X}$ point in some periods.

The experimented AEPC-1 - AEPC-4 have earlier been studied by TalTech research group to detect the pulse wave through EBI measurements. The effectiveness of selected AEPCs was determined by FEM-modelling through finding the emerging sensitivity distributions - the presence of positive and negative regions [13]. The simulation showed the highest sensitivity ratio (and the best result among the four chosen AEPC's in current study) in the case of AEPC-3 [13]. The result depends on the distribution of sensitivities i.e., is largely affected by the complex and layered structure of thorax with its vastly varying conductivity. Based on the simulations it has been claimed that most of the contribution for ICG comes from the skeletal muscle and upper thorax and less than $1 \%$ from the aorta [14]. While the representation of the exact sensitivity distribution in human body is not yet found, the controversy remains - and the gold standard for monitoring the stroke volume is not defined.

\section{CONCLUSions And Future Work}

The performed experimentation confirmed that the utilization of coinciding electrode locations for simultaneous determination of ECG and ICG is highly feasible. Moreover, the variety of electrode locations on thorax and arm provide waveforms, where the hemodynamic pattern is present in quality, which is enough to determine the important feature points and time intervals. The next research question is improving the feature extraction algorithm for X-point and finding other corrections. Additionally, to improve the credibility of the results, the next step is to get more statistical data from various persons under different conditions is important.

\section{ACKNOWLEDGMENT}

The authors would like to thank colleagues prof M. Min, dr A. Krivošei and dr M. Rist for their valuable help.

\section{REFERENCES}

[1] O. G. Martinsen and S. Grimnes, Bioimpedance and Bioelectricity Basics, 3rd ed. Academic Press, Aug 2014.

[2] P. S. Raj and D. Hatzinakos, "Feasibility of single-arm single-lead ecg biometrics," in 2014 22nd European Signal Processing Conference (EUSIPCO), 2014, pp. 2525-2529.

[3] H. Yazdanian, A. Mahnam, M. Edrisi, and M. Esfahani, "Design and implementation of a portable impedance cardiography system for noninvasive stroke volume monitoring," Journal of Medical Signals and Sensors, vol. 6, pp. 47-56, 012016.

[4] G. Cybulski, Ambulatory Impedance Cardiography - The Systems and their Applications. Springer, 2011.

[5] O. J. Escalona, L. McFrederick, M. Borges, P. Linares, R. Villegas, G. I. Perpiñan, J. McLaughlin, and D. McEneaney, "Wrist and arm body surface bipolar ecg leads signal and sensor study for long-term rhythm monitoring," in 2017 Computing in Cardiology (CinC), 2017, pp. 1-4.

[6] S. Gonçalves and R. Carneiro Martins, "Non-contact wearable single forearm cardiac biopotential acquisition device," Journal of Physics Conference Series, vol. 459, pp. 2065-, 092013.

[7] A. Hafid, S. Benouar, M. Kedir-Talha, M. Attari, and F. Seoane, "Simultaneous recording of icg and ecg using Z-rpi device with minimum number of electrodes," Journal of Sensors, vol. 2018, pp. $1-7,112018$.

[8] L. N. Harrold and A. R. Diciaccio, "Icg/ecg monitoring apparatus,",", U.S. Patent Application 20120323 106A1, Dec. 20, 2012.

[9] M. Nakagawara and K. Yamakoshi, "A portable instrument for noninvasive monitoring of beat-by-beat cardiovascular haemodynamic parameters based on the volume-compensation and electrical-admittance method," Med Biol Eng Comput., vol. 38, no. 1, pp. 17-25, 2000.

[10] HF2 User Manual - ziControl Edition, Zurich Instruments, 2019.

[11] B. B. Sramek, "Status report on bomed's electrical bioimpedance," in Proceedings of the Annual International Conference of the IEEE Engineering in Medicine and Biology Society, 1988, pp. 51 vol.1-.

[12] S. M. M. Naidu, U. R. Bagal, P. C. Pandey, S. Hardas, and N. D. Khambete, "Detection of characteristic points of impedance cardiogram and validation using doppler echocardiography," in 2014 Annual IEEE India Conference (INDICON), 2014, pp. 1-6.

[13] K. Pesti, M. Metshein, P. Annus, H. Kõiv, and M. Min, "Electrode placement strategies for the measurement of radial artery bioimpedance: Simulations and experiments," IEEE Transactions on Instrumentation and Measurement, vol. 70, pp. 1-10, 2021.

[14] R. P. Patterson, "Fundamentals of impedance cardiography," IEEE Engineering in Medicine and Biology Magazine, vol. 8, no. 1, pp. 35-38, 1989. 